Stud. Univ. Babeş-Bolyai Math. 65(2020), No. 4, 641-649

DOI: $10.24193 /$ subbmath.2020.4.10

\title{
Gradient-type deformations of cycles in EPH geometries
}

\author{
Mircea Crasmareanu
}

\begin{abstract}
The aim of this paper is to study the cycles of EPH geometries through their homogeneous gradient-type deformations recently introduced by the author. A special topic is the orthogonality between a given cycle $C$ and its deformations as well as between $C$ and its rotated version $R(C)$.
\end{abstract}

Mathematics Subject Classification (2010): 51N25, 51M09, 53A40.

Keywords: EPH geometries, cycle, deformation, orthogonality, rotation.

\section{Introduction}

It is well-known that up to isomorphisms there are three 2-dimensional real algebras: $\mathbb{C}=\mathbb{R}[X] /\left(x^{2}+1\right), \mathbb{D}=\mathbb{R}[X] /\left(x^{2}\right)$ and $\mathbb{A}=\mathbb{R}[X] /\left(x^{2}-1\right)$. The theory of the first algebra is richer than the following two, a fact corresponding to the field property of $\mathbb{C}$. Inspired by the terminology of $[6$, p. 1458$]$ or $[7$, p. 2] we call $E P H$ geometries these spaces and a common image consists in $A(\sigma):=\mathbb{R}[X] /\left(x^{2}-\sigma\right)$ with $\sigma:=i^{2} \in\{-1,0,1\}$ respectively and $i$ the corresponding imaginary unit.

The recent papers [2] and [5], devoted to Finsler geometry, start with a deformation of a conic $\Gamma$ obtained by deforming the gradient vector field for the quadratic form defining $\Gamma$. These deformations are inspired by the scaling (linear) transformation of Computer Graphics: $(x, y) \in \mathbb{R}^{2} \rightarrow\left(\lambda_{x} \cdot x, \lambda_{y} \cdot y\right) \in \mathbb{R}^{2}$, following [8, p. 136]. Using the well-known invariants from the Euclidean geometry of conics we obtained the classifications of the new conics which depends on two scalars denoted $\alpha$ and $\beta$, having the role of $\lambda_{x}, \lambda_{y}$. The new conic of [2], denoted $\tilde{\Gamma}$, is a degenerate one and we could interpret the map $\Gamma \rightarrow \tilde{\Gamma}$ as a "curve shortening" transformation. The same fact holds for the new conic of [5], denoted $\Gamma^{m}$, if the initial conic $\Gamma$ does not have linear terms.

In this note we use these classes of gradient-type deformation to a main object of EPH geometries, called cycle, which is a particular case of conic sections, invariant under the action of the group $S L(2, \mathbb{R})$ through Mobiüs transformations. A detailed 
analysis of the deformed cycles depends on the vanishing or not of $\sigma$ as well as the vanishing or not of a parameter $k$ separating the circles to lines. Also, we discuss the transformation of a square matrix associated to any cycle $C$.

Moreover, we treat these deformations in terms of $A(\sigma)$-numbers. In the second section we study the orthogonality of a given cycle $C$ with its deformations restricting to the $\sigma \neq 0$ case. In the last section we introduce a natural rotation $R$ in $A(\sigma)$ and we study the relationships between a given $C$ and its rotated cycle $R(C)$.

\section{The cycles of EPH geometries and their gradient-type deformations}

In the two-dimensional Euclidean space $\mathbb{R}^{2}$ let us consider the conic $\Gamma$ implicitly defined by $f \in C^{\infty}\left(\mathbb{R}^{2}\right)$ as: $\Gamma=\left\{(x, y) \in \mathbb{R}^{2} \mid f(x, y)=0\right\}$ where $f$ is a quadratic function of the form $f(x, y)=r_{11} x^{2}+2 r_{12} x y+r_{22} y^{2}+2 r_{10} x+2 r_{20} y+r_{00}$ with $r_{11}^{2}+r_{12}^{2}+r_{22}^{2} \neq 0$ for the non-degenerate conics.

It is well-known that the gradient vector field of $f$, namely

$$
\nabla f=\left(f_{x}=\frac{\partial f}{\partial x}, f_{y}=\frac{\partial f}{\partial y}\right),
$$

gives important properties of $\Gamma$; for example, the centers of $\Gamma$ are exactly the critical points of $\nabla f$. Inspired by this fact we introduced recently:

Definition 2.1. Fix the scalars $\alpha, \beta$ with $\alpha \beta \neq 0$.

i) $([2$, p. $86-87],[3$, p. 60$])$ The $(\alpha, \beta)$-deformation of $\Gamma$ is the conic:

$$
\tilde{\Gamma}=\Gamma_{\alpha, \beta}: \alpha\left[\frac{1}{2} f_{x}\right]^{2}+\beta\left[\frac{1}{2} f_{y}\right]^{2}=0 .
$$

ii) $([5$, p. 102]) The $(\alpha, \beta)$-mixed deformation of $\Gamma$ is the conic:

$$
\Gamma^{m}=\Gamma_{\alpha, \beta}^{m}: \alpha y\left[\frac{1}{2} f_{x}\right]+\beta x\left[\frac{1}{2} f_{y}\right]=0 .
$$

A main object in EPH geometries is given in [6, p. 1459], [7, p. 4]:

Definition 2.2. The common name cycle will be used to denote circles, parabolas and hyperbolas (as well as straight lines as their limits) in the respective EPH geometry.

An analytical study of a cycle can be done via the general equation given in $[6, \mathrm{p}$. 1460] or $[7$, p. 6]:

$$
C: f(u, v):=k\left(u^{2}-\sigma v^{2}\right)-2 l u-2 n v+m=0
$$

and hence $C$ is a conic section completely defined by the data $(k, l, n, m) \in \mathbb{P}^{3}$. As usual, if $k=0$ then $C$ can be called a degenerate cycle. In fact, in the cited works $C$ is identified with the matrix:

$$
C_{\breve{\sigma}}^{s}:=\left(\begin{array}{cc}
l+\breve{1} s n & -m \\
k & -l+\breve{1} s n
\end{array}\right)
$$


where $s$ is a new parameter, usually equal to \pm 1 , and a new imaginary unit $\breve{1}$. Its square $\breve{\sigma}:=\breve{1}^{2}$ belongs again to $\{-1,0,1\}$ but independently of $\sigma$.

Since $C$ is a conic section we can apply the ideas of Definition 2.1 to introduce the gradient-type deformations of a cycle:

$$
\left\{\begin{array}{l}
\tilde{C}=C_{\alpha, \beta}: \alpha(k u-l)^{2}+\beta(k \sigma v+n)^{2}=0 \\
C^{m}: \alpha v(k u-l)-\beta u(k \sigma v+n)=0
\end{array}\right.
$$

which yields immediately:

Proposition 2.3. Since $\alpha \neq 0$ we have:

i) $\tilde{C}$ is a cycle if and only if $\sigma(\alpha+\sigma \beta)=0$,

ii) $C^{m}$ is a cycle if and only if $k(\alpha-\beta \sigma)=0$. In this case $C^{m}$ is the straight line:

$$
(\beta n) u+(\alpha l) v=0 .
$$

Example 2.4. In the following we discuss the remarkable particular cases of the result above.

i) Suppose $\sigma=0$. Then $\tilde{C}$ is the cycle:

$$
\tilde{C}:(k u-l)^{2}+\frac{\beta}{\alpha} n^{2}=0
$$

with the matrix:

$$
\tilde{C}_{\breve{\sigma}}^{s}=\left(\begin{array}{cc}
k l & -\left(l^{2}+\frac{\beta}{\alpha} n^{2}\right) \\
k^{2} & -k l
\end{array}\right) .
$$

The degenerate case of an initial line i.e. $k=0$ is possible if and only if $\alpha l^{2}+\beta n^{2}=0$ which is relation (2.19) below. If $k \neq 0$ then, due to the projective character of the coefficients of a cycle, we get the matrix:

$$
\tilde{C}_{\breve{\sigma}}^{s}=\left(\begin{array}{cc}
l & -\frac{1}{k}\left(l^{2}+\frac{\beta}{\alpha} n^{2}\right) \\
k & -l
\end{array}\right) .
$$

If $\frac{\beta}{\alpha}>0$ then $\tilde{C}$ is a void set for $n \neq 0$ while $n=0$ gives the deformation:

$$
C: k u^{2}-2 l u+m=0 \rightarrow \tilde{C}: k u=l(\text { line }: k \neq 0) .
$$

If $\frac{\beta}{\alpha}<0$ then we have the lines:

$$
\tilde{C}: k u-l= \pm \sqrt{-\frac{\beta}{\alpha}} n .
$$

$C^{m}$ is a cycle if and only if $k=0$ which means that we have the mixed deformation:

$$
C: 2 l u+2 n v-m=0(\text { line }) \rightarrow C^{m}:(\beta n) u+(\alpha l) v=0 \text { (line). }
$$

If $\beta=-\alpha$ then these two lines are Euclidean orthogonal. From the matrix point of view the deformation (2.11) means:

$$
C_{\breve{\sigma}}^{s}=\left(\begin{array}{cc}
l+\breve{1} s n & -m \\
0 & -l+\breve{1} s n
\end{array}\right) \rightarrow C_{\breve{\sigma}}^{m, s}=\left(\begin{array}{cc}
-\beta n+\breve{1} s(-\alpha l) & 0 \\
0 & \beta n+\breve{1} s(-\alpha l)
\end{array}\right) .
$$

ii) For $\sigma \neq 0$ we have that $\tilde{C}$ is a cycle only for $\beta=-\frac{\alpha}{\sigma}=-\sigma \alpha$ and then:

$$
\tilde{C}:\left[k(u+i v)-l+\frac{n}{i}\right]\left[k(u-i v)-l-\frac{n}{i}\right]=0 .
$$


Hence, if $k \neq 0$ then $\tilde{C}$ consists in a single point: $M=\left(\frac{l}{k},-\frac{n}{k \sigma}\right)$. Let us point out that for $\sigma \neq 0$ we have $\frac{1}{\sigma}=\sigma$ and hence $M=\left(\frac{l}{k},-\sigma \frac{n}{k}\right)$ which is exactly the $e / h$-center of the initial cycle $C$, as it is introduced in formula $(7)$ of $[6$, p. 1460] or $[7$, p. 7]. In conclusion, for $\sigma \cdot k \neq 0$ we have the deformation:

$$
C \rightarrow \tilde{C}=\text { its center. }
$$

The matrix corresponding to $\tilde{C}$ is:

$$
\tilde{C}_{\breve{\sigma}}^{s}=\left(\begin{array}{cc}
k(l+\breve{1} s n) & n^{2} \sigma-l^{2} \\
k^{2} & k(-l+\breve{1} s n)
\end{array}\right)
$$

which for $k=0$ becomes:

$$
\tilde{C}_{\breve{\sigma}}^{s}=\left(\begin{array}{cc}
0 & n^{2} \sigma-l^{2} \\
0 & 0
\end{array}\right)
$$

while for $k \neq 0$, due to the projective character of the parameters of a cycle:

$$
\tilde{C}_{\breve{\sigma}}^{s}=\left(\begin{array}{cc}
l+\breve{1} s n & \frac{1}{k}\left(n^{2} \sigma-l^{2}\right) \\
k & -l+\breve{1} s n
\end{array}\right) .
$$

The same case $\sigma \cdot k \neq 0$ for ii) of proposition above gives $\beta=\frac{\alpha}{\sigma}=\sigma \alpha$ and $C^{m}$ is the line:

$$
C^{m}: n u+(\sigma l) v=0 .
$$

For elliptic geometry the condition $\beta=-\frac{\alpha}{\sigma}=-\sigma \alpha$ becomes the equality $\alpha=\beta$ discussed in [2, p. 89] and [3, p. 62]; it can be called the diagonal case. Remark that the elliptic center $\tilde{C}$ of $(2.14)$ is obtained in [6, p. 1461] or $[7$, p. 8] from the vanishing condition $\operatorname{det} C_{-1}^{s}=0$.

Remark 2.5. The cycle $C^{m}$ contains the origin $(u, v)=(0,0)=O$. This fact holds for $\tilde{C}$ if and only if:

$$
\alpha l^{2}+\beta n^{2}=0 .
$$

With the discussion of above particular cases it results:

i) for $\sigma=0$ the only available case is $\frac{\beta}{\alpha}<0$ yielding:

$$
l_{ \pm}= \pm \sqrt{-\frac{\beta}{\alpha}} n \text {. }
$$

ii) for $\sigma \neq 0$ since $\beta=-\frac{\alpha}{\sigma}=-\sigma \alpha$ we get that for the elliptic geometry the only possible case is $O=M$ the center of $C$ while for the hyperbolic geometry:

$$
l_{ \pm}= \pm n \text {. }
$$

The gradient-type deformation of a standard (i.e. Euclidean) ellipse is discussed in example $2.2 \mathrm{i}$ ) of $[2$, p. 87]. Let us point out that (2.20) and (2.21) coincide for $\beta=-\alpha$ which for the case ii) correspond to the hyperbolic geometry. Hence the above cases i) and ii) are completely different, both from $\sigma$ and the sign of $\frac{\beta}{\alpha}$ points of view.

Returning to the general case of $\alpha$ and $\beta$ we treat the considered deformations within $A(\sigma)$ following the model of [3] and [5]. More precisely, with the usual notation $z=u+i v \in A(\sigma)$ we derive the expression of $C$ :

$$
C: F(z, \bar{z}):=k z \bar{z}+B z+\bar{B} \bar{z}+m=0, \quad B:=-l-\frac{n}{\sigma} i \in A(\sigma)(\sigma \neq 0) .
$$


For $\sigma=0$ we have: $B=-l-\frac{n}{i}$. The inverse relationship between $f$ and $F$ is:

$$
l=-\Re B, \quad n=-\sigma \Im B
$$

with $\Re$ and $\Im$ respectively the real and imaginary part. By replacing in (2.5) the usual relations:

we get:

$$
u=\frac{1}{2}(z+\bar{z}), \quad v=\frac{1}{2 i}(z-\bar{z})
$$

$$
\left\{\begin{array}{l}
\tilde{C}: \alpha[k(z+\bar{z})-2 l]^{2}+\beta[k i(z-\bar{z})+2 n]^{2}=0 \\
C^{m}: \alpha(z-\bar{z})[k(z+\bar{z})-2 l]-\beta(z+\bar{z})[k \sigma(z-\bar{z})+2 n i]=0
\end{array}\right.
$$

For the case $\sigma \neq 0$ we follow the discussion of Example 2.4ii and then:

$$
\left\{\begin{array}{l}
\tilde{C}:[k(z+\bar{z})-2 l]^{2}-\sigma[k i(z-\bar{z})+2 n]^{2}=0, \\
C^{m}:(z-\bar{z})[k(z+\bar{z})-2 l]-\sigma(z+\bar{z})[k \sigma(z-\bar{z})+2 n i]=0 .
\end{array}\right.
$$

The second equation (2.26) reduces to:

$$
C^{m}: B z-\bar{B} \bar{z}=0 \leftrightarrow B z \in \mathbb{R}
$$

and hence, for $B \neq 0$ we have the line: $z=\bar{B} \cdot \mathbb{R}$.

We finish this section by applying to the cycle $C$ (not containing the origin, hence $m \neq 0$ ) the inversion $J: z \in A(\sigma)^{*} \rightarrow \frac{1}{z}=w$. We get a new cycle, expressed in $w$ :

$$
J(C): m w \bar{w}+\bar{B} w+B \bar{w}+k=0
$$

which means $J:(k, l, n, m) \rightarrow(m, l,-n, k)$. With (2.26)-(2.27) its gradient deformations for $\sigma \neq 0$ are:

$$
\left\{\begin{array}{l}
\widetilde{J(C)}:[m(w+\bar{w})-2 l]^{2}-\sigma[m i(w-\bar{w})-2 n]^{2}=0, \\
J(C)^{m}: B \bar{w}-\bar{B} w=0 \leftrightarrow \bar{B} w \in \mathbb{R} .
\end{array}\right.
$$

Again, if $B \neq 0$ then the second cycle from from above is the line: $w=B \cdot \mathbb{R}$.

\section{Orthogonality in the geometry of cycles}

In [6, p. 1462] or [7, p. 2] a Möbius-invariant (indefinite) inner product (depending on $\breve{\sigma}$ ) is defined on the set of cycles through:

$$
<C_{\breve{\sigma}}^{s}, \hat{C}_{\breve{\sigma}}^{s}>:=\operatorname{Tr}\left(C_{\breve{\sigma}}^{s} \cdot{\overline{\hat{C}_{\breve{\sigma}}^{s}}}^{s}\right)
$$

which yields an associated $\breve{\sigma}$-orthogonality. Here, the bar means the conjugation with respect to $\breve{~}$.

For our setting we derive firstly the norms of a cycle and its gradient-type deformations for $k \sigma \neq 0$ :

$$
\left\{\begin{array}{l}
\left\|C_{\breve{\sigma}}^{s}\right\|^{2}=2\left(l^{2}-k m-\breve{\sigma} n^{2}\right)=\left\|J(C)_{\breve{\sigma}}^{s}\right\|^{2}, \\
\left\|\tilde{C}_{\breve{\sigma}}^{s}\right\|^{2}=2(\sigma-\breve{\sigma}) n^{2}, \quad\left\|C_{\breve{\sigma}}^{m, s}\right\|^{2}=\frac{1}{2}\left(n^{2}-\breve{\sigma} l^{2}\right) .
\end{array}\right.
$$

Let us remark that:

$$
\operatorname{det} C_{\breve{\sigma}}^{s}=k m+\breve{\sigma} n^{2}-l^{2} \rightarrow\left\|C_{\breve{\sigma}}^{s}\right\|^{2}=\left\|J(C)_{\breve{\sigma}}^{s}\right\|^{2}=-2 \operatorname{det} C_{\breve{\sigma}}^{s} .
$$

Secondly, we study all the possible cases of orthogonality for our setting: 
Theorem 3.1. Let $\sigma \neq 0$ and the cycle $C$ with $k \neq 0$. Then:

1) $C$ is $\breve{\sigma}$-orthogonal to its gradient deformation $\tilde{C}$ if and only if:

$$
l^{2}-k m+(\sigma-2 \breve{\sigma}) n^{2}=0 .
$$

2) $C$ is $\breve{\sigma}$-orthogonal to its mixed-gradient deformation $C^{m}$ if and only if:

$$
(1-\sigma \breve{\sigma}) n l=0 .
$$

3) $\tilde{C}$ is $\breve{\sigma}$-orthogonal to $C^{m}$ if and only if (3.4) holds.

4) Suppose also $m \neq 0$. Then $C$ is $\breve{\sigma}$-orthogonal to $J(C)$ if and only if:

$$
2\left(l^{2}+\breve{\sigma} n^{2}\right)-k^{2}-m^{2}=0 .
$$

Proof. 1) A straightforward computation gives:

$$
<C_{\breve{\sigma}}^{s}, \tilde{C}_{\breve{\sigma}}^{s}>=l^{2}-k m+(\sigma-2 \breve{\sigma}) n^{2} .
$$

2) The matrix of $C^{m}$ from (2.18) is:

$$
C_{\breve{\sigma}}^{m, s}=\frac{1}{2}\left(\begin{array}{cc}
n+\breve{1} s \sigma l & 0 \\
0 & -n+\breve{1} s \sigma l
\end{array}\right)
$$

and then:

$$
<C_{\breve{\sigma}}^{s}, C_{\breve{\sigma}}^{m, s}>=(1-\sigma \breve{\sigma}) n l
$$

3) The same computation as above.

4) The matrix of $J(C)$ is:

$$
J(C)_{\breve{\sigma}}^{s}:=\left(\begin{array}{cc}
l-\breve{1} s n & -k \\
m & -l-\breve{1} s n
\end{array}\right)
$$

and:

$$
<C_{\breve{\sigma}}^{s}, J C_{\breve{\sigma}}^{s}>=2\left(l^{2}+\breve{\sigma} n^{2}\right)-m^{2}-k^{2} .
$$

which gives the conclusion.

Example 3.2. Suppose $\sigma=\breve{\sigma}$. Then $1-\sigma \breve{\sigma}=0$ since $\sigma^{2}=1$ and then $C^{m}$ is both orthogonally on $C$ and $\tilde{C}$. In this case $C$ is orthogonally to $\tilde{C}$ if and only if $l^{2}-k m-\breve{\sigma} n^{2}=0$ but from the first equation (3.2) this means that $\|C\|=0$ i.e. $C$ is also self-orthogonal.

Returning to the Möbius-type study of cycles we continue this section considering some transformation of cycles. The first one is inspired by [1, p. 2706]. Let $\alpha \in A(\sigma)$ with module $|\alpha| \neq 1$ and consider the map $T_{\alpha}: A(\sigma) \rightarrow A(\sigma)$ :

$$
T_{\alpha}(z)=z+\alpha \bar{z}:=w .
$$

It follows directly that $T_{\alpha}$ is a bijective map with the inverse:

$$
z:=T_{\alpha}^{-1}(w)=\frac{1}{1-|\alpha|^{2}}(w-\alpha \bar{w}) .
$$

Replacing this expression of $z$ in $(2.22)$ we find the image of cycle $C$ through $T_{\alpha}$ :

$$
T_{\alpha}(C): k|w-\alpha \bar{w}|^{2}+\left(1-|\alpha|^{2}\right)\left[(B-\bar{\alpha} \bar{B}) w+(\bar{B}-\alpha B) \bar{w}+\left(1-|\alpha|^{2}\right) m\right]=0
$$

but this curve is not a cycle for $\alpha \cdot k \neq 0$. 
The second transformation is a Blaschke factor $B_{a}$ defined by $a \in A(\sigma)$ with module $|a|<1$ :

$$
w:=B_{a}(z)=\frac{z-a}{1-\bar{a} z}
$$

having the inverse:

$$
z=B_{-a}(w)=\frac{w+a}{1+\bar{a} w} .
$$

The Blaschke transformation of the cycle (2.22) is again a cycle:

$$
B_{a}(C): b_{a}(k) w \bar{w}+b_{a}(B) w+\overline{b_{a}(B)} \bar{w}+b_{a}(m)=0
$$

with:

$$
\left\{\begin{array}{l}
b_{a}(k)=k+m|a|^{2}+2 \Re(B a), \\
b_{a}(B)=(k+m) \bar{a}+B+\bar{B} \bar{a}^{2}, \\
b_{a}(m)=m+k|a|^{2}+2 \Re(B a) .
\end{array}\right.
$$

Example 3.3. Suppose that $|B|<1$ and let $a=\bar{B}$. Then the Blaschke transformation of the coefficients is:

$$
\left\{\begin{array}{l}
b_{\bar{B}}(k)=k+(m+2)|B|^{2}, \\
b_{\bar{B}}(B)=\left(k+m+1+|B|^{2}\right) B, \\
b_{\bar{B}}(m)=m+(k+2)|B|^{2} .
\end{array}\right.
$$

The last transformation is a similarity defined by $a, b \in A(\sigma)$ with $a \neq 0$ :

$$
w:=S_{a, b}(z)=a z+b,
$$

having the inverse:

$$
z=\frac{1}{a}(w-b)=S_{\frac{1}{a}, \frac{-b}{a}}(w) .
$$

The similarity transformation of the cycle (2.22) is again a cycle:

$$
S_{a, b}(C): k w \bar{w}+(B \bar{a}-k \bar{b}) w+(\bar{B} a-k b) \bar{w}+m|a|^{2}+k|b|^{2}-2 \Re(B b \bar{a})=0 .
$$

If the initial cycle $C$ is non-degenerate then we restrict to the case $k=1$ due to the projective character of the coefficients of $C$. Then a non-degenerate $C$ is called decomposable if it is a product of lines:

$$
C:(z-B)(\bar{z}-\bar{B})=0
$$

which means that $m=|B|^{2}=l^{2}-\sigma n^{2}$. A similarity preserves the class of decomposable cycles since its image is:

$$
S_{a, b}(C):(w-b+a \bar{B})(\bar{w}-\bar{b}+\bar{a} B) .
$$

From (3.3) it follows that a decomposable cycle has:

$$
\operatorname{det} C_{\breve{\sigma}}^{s}=(\breve{\sigma}-\sigma) n^{2} .
$$




\section{The rotation of a cycle}

In this section we suppose that $\sigma \neq 0$. In $A(\sigma)$ we introduce the rotation map $R:(u, v) \rightarrow i \cdot(u, v)=(\sigma v, u)$; then its square is: $R^{2}=\sigma I$. It follows that a given cycle $C$ has an associated rotation cycle $R(C)$ with equation:

$$
R(C): k\left(\sigma^{2} v^{2}-\sigma u^{2}\right)-2 l \sigma v-2 n u+m=0 .
$$

A short computation gives a more simple form:

$$
R(C): k\left(u^{2}-\sigma v^{2}\right)+2(\sigma n) u+2 l v-\sigma m=0
$$

and then we have the deformation:

$$
C=(k, l, n, m) \rightarrow R(C)=(k,-\sigma n,-l,-\sigma m) .
$$

The general rotation of conics is treated in [4].

Remark 4.1. Concerning the compositions $J \circ R$ and $R \circ J$ we have:

$$
J \circ R(C)=(-\sigma m,-\sigma n, l, k), \quad R \circ J(C)=(m, \sigma n,-l,-\sigma k)
$$

and then $J$ and $R$ anti-commutes in the hyperbolic setting respectively $J$ and $R$ commutes if and only if $l=0$ in the complex setting: $\sigma=-1$.

In terms of associated matrix we have:

$$
R(C)_{\breve{\sigma}}^{s}=\left(\begin{array}{cc}
-\sigma n-\breve{1} s l & \sigma m \\
k & \sigma n-\breve{1} s l
\end{array}\right),\left\|R(C)_{\breve{\sigma}}^{s}\right\|^{2}=2\left(n^{2}+\breve{\sigma} l^{2}+\sigma k m\right) .
$$

Then $R$ preserves the norm of $C$ if and only if:

$$
(\sigma+1) k m+(\breve{\sigma}-1) l^{2}+(1-\breve{\sigma}) n^{2}=0 .
$$

Also, recall from section 2 that the $e / h$-center of $C$ is $M\left(\frac{l}{k},-\sigma \frac{n}{k}\right)$ and hence its rotation is $R(M)=\left(-\frac{n}{k}, \frac{l}{k}\right)$. But the center of $R(C)$ is $\bar{M}=\left(-\frac{\sigma n}{k}, \frac{\sigma l}{k}\right)$ and then $\bar{M}=\sigma R(M)$; these points coincide for $\sigma=1$. have:

Concerning the orthogonality of this new cycle with the previous three cycles we

Proposition 4.2. Let $C$ be a cycle with $k \neq 0$. Then:

i) $C$ is $\breve{\sigma}$-orthogonal to its rotated cycle $R(C)$ if and only if:

$$
(\breve{\sigma}-\sigma) n l+(\sigma-1) k m=0 .
$$

ii) $\tilde{C}$ is $\breve{\sigma}$-orthogonal to $R(C)$ if and only if:

$$
2(\breve{\sigma}-\sigma) n l+\sigma\left(n^{2}+k m\right)-l^{2}=0 .
$$

iii) $C^{m}$ is $\breve{\sigma}$-orthogonal to $R(C)$ if and only if:

$$
\breve{\sigma} l^{2}=n^{2} .
$$

Proof. A straightforward computation gives:

$$
\begin{gathered}
<C_{\breve{\sigma}}^{s}, R(C)_{\breve{\sigma}}^{s}>=2[(\breve{\sigma}-\sigma) n l+(\sigma-1) k m], \\
<\tilde{C}_{\breve{\sigma}}^{s}, R(C)_{\breve{\sigma}}^{s}>=2(\breve{\sigma}-\sigma) n l+\sigma\left(n^{2}+k m\right)-l^{2} \\
<C_{\breve{\sigma}}^{m, s}, R(C)_{\breve{\sigma}}^{s}>=2 \sigma\left(\breve{\sigma} l^{2}-n^{2}\right]
\end{gathered}
$$

which yields the conclusion. 
Example 4.3. Suppose that $\sigma=\breve{\sigma}=1$. Then $R(C)$ is orthogonal to $C$ and:

a) is orthogonal to $\tilde{C}$ if and only if: $l^{2}=n^{2}+k m$; for $k=1$ this means that $C$ is decomposable,

b) is orthogonal to $C^{m}$ if and only if: $l_{ \pm}= \pm n$, which is exactly the relation (2.21).

\section{References}

[1] Chuaqui, M., Duren, P., Osgood, B., Ellipses, near ellipses, and harmonic Möbius transformations, Proc. Am. Math. Soc., 133(2005), no. 9, 2705-2710.

[2] Crasmareanu, M., A gradient-type deformation of conics and a class of Finslerian flows, An. Ştiinţ. Univ. Ovidius Constanţa, Ser. Mat., 25(2017), no. 2, 85-99.

[3] Crasmareanu, M., A complex approach to the gradient-type deformations of conics, Bull. Transilv. Univ. Braşov, Ser. III, Math. Inform. Phys., 10(59)(2017), no. 2, 59-62.

[4] Crasmareanu, M., From rotation of conics to a class of Finslerian flows, Annals Univ. Craiova Ser. Mat. Inf., 45(2018), no. 2, 275-282.

[5] Crasmareanu, M., A mixed gradient-type deformation of conics and a class of FinslerianRiemannian flows, An. Univ. Oradea Fasc. Mat., 26(2019), no. 1, 101-107.

[6] Kisil, V.V., Starting with the group $S L_{2}(\mathbb{R})$, Notices Am. Math. Soc., 54(2007), no. 11, 1458-1465.

[7] Kisil, V.V., Geometry of Möbius Transformations. Elliptic, Parabolic and Hyperbolic Actions of $S L_{2}(\mathbb{R})$, Hackensack, NJ: World Scientific, 2012.

[8] Rovenski, V., Modeling of Curves and Surfaces with MATLAB, Springer Undergraduate Texts in Mathematics and Technology, Springer Berlin, 2010.

Mircea Crasmareanu

Faculty of Mathematics, University "Al. I.Cuza", Iaşi, 700506, Romania

e-mail: mcrasm@uaic.ro 